\title{
Interferometric orbit determination for geostationary satellites
}

\author{
Roger M. FUSTER*, Marc Fernández USÓN \& Antoni Broquetas IBARS \\ UPC, Dept. TSC, Remote Sensing Lab. C/ Jordi Girona [1-3], Barcelona 08034, Spain \\ Received $\operatorname{xxxxxxxx} \mathrm{xx}, \mathrm{xxxx}$; accepted $\operatorname{xxxxxxxx} \mathrm{xx}, \mathrm{xxxx}$
}

\begin{abstract}
One of the main challenges in GeoSAR processing is accurately determining the satellite orbit. To tackle this challenge, a multiple baseline ground-based interferometer is proposed. As a proof of concept, this paper presents the results obtained from a single baseline prototype, whose results can be extrapolated to a larger system.
\end{abstract}

Keywords GeoSAR, interferometry, orbit determination, geostationary SAR

Citation Fuster R M, Usn M F, Ibars A B. Interferometric orbit determination for geostationary satellites. Sci China Inf Sci, 2017, 60(1): xxxxxx, doi: 10.1007/s11432-016-9052-y

\section{Introduction: the GeoSAR mission}

A variety of applications, including land stability control and monitoring natural hazards such as volcanic activity or earthquakes would substantially benefit from permanent radar monitoring, as the fast evolution of these hazards is not observable with current low Earth orbit (LEO) based systems. To overcome this drawback, GeoSAR missions were proposed.

There are two main approaches regarding GeoSAR missions. On the one hand, the use of platforms on geosynchronous orbits has been recently studied [1]. On the other hand, recent studies have shown the possibility to operate a radar payload hosted by a communication satellite in a geostationary orbit [2]. The movement of the satellite in the orbit does not follow a perfect equatorial trajectory, but has a slight eccentricity and inclination that can be used to form the synthetic aperture required to obtain images. $\equiv$ work focuses on the second approach. A proper comparison between geosynchronous and geostationary SAR is discussed in [3].

Several sources affect the along-track phase history in GeoSAR missions, causing unwanted fluctuations that may result in image defocusing. One main expected contributor to azimuth phase noise are orbit determination errors. An accurate image of the scene after SAR processing can be obtained if the range history of every point of the scene is accurately known. This fact necessitates high-precision orbit modeling (with accuracies in the order of magnitude of $\lambda$ ), the use of suitable techniques for atmospheric phase screen compensation [4], and the study and correction of ionospheric effects [5], especially at large wavelengths such as the L band. Such orbital determination requirements are well beyond the usual

*Corresponding author (email: r.martin.fuster@gmail.com) 


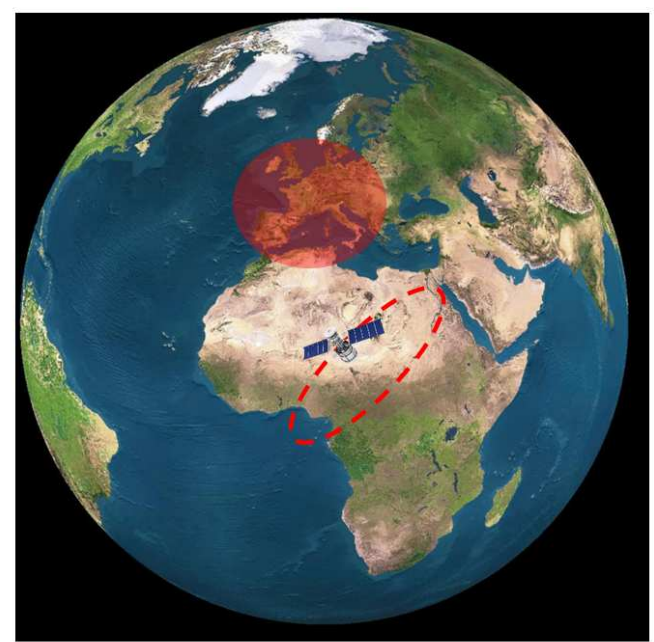

Figure 1 (Color online) Conceptual image of the coverage and geometry of a GeoSAR mission.

systems used to manage repositioning of satellites in geostationary orbits. To achieve an accurate satellite orbit, two possible precise systems have been discussed in [6].

The first approach involves the deployment of several active radar calibrators (ARCs) over Europe that would be used to focus the radar images. While this approach is conceptually solid and requires no synchronization between transceivers, it demands the calibrators to be located on the area to be observed, and cannot be properly tested before the radar payload is launched in the orbit.

The second approach based on interferometry is of a higher complexity owing to the synchronization of the receivers; however, the interferometer can be tested as an orbital determination device with actual geostationary communication satellites. In addition, it can be placed regardless of the area to be observed by the radar, as long as the interferometer receives enough power from the satellite.

This work focuses on the second approach, presenting the initial results obtained by using a groundbased interferometry system. The document is organized as follows. First, we provide some background on interferometry and explain the main system configuration used to obtain raw positioning data. Second, we explain the least-squares technique for orbit determination adopted to obtain orbital parameters from the interferometric phase. Finally, the results achieved are analyzed and discussed.

\section{Theoretical background}

The principle of interferometry techniques is to coherently combine signals generated from the same source at different locations so that information about the source can be obtained. These techniques generate an interference signal by means of either superposition in traditional optical interferometry [7], or correlation as in modern radio-interferometry [8].

The basic interferometer setup consists of an emitter source and at least two receivers. Each receiver pair is entitled to obtain an interference signal and its performance will greatly depend on the separation between receivers, commonly known as baseline.

\subsection{Correlation interferometry}

The baseband equivalent of the arriving signals at each receiving antenna can be approximately defined as the transmitted complex signal affected by amplitude and phase components, caused by the transmission path among other factors.

$$
\begin{gathered}
T x(t)=|T x(t)| \mathrm{e}^{\mathrm{j} \measuredangle T x(t)}, \\
R x_{i}(t)=A_{i} T x\left(t-t_{i}\right) \mathrm{e}^{-\mathrm{j} \omega t_{i}}+\omega_{i}(t)=A_{i} T x\left(t-t_{i}\right) \mathrm{e}^{-\mathrm{j} \alpha_{i}}+\omega_{i}(t),
\end{gathered}
$$




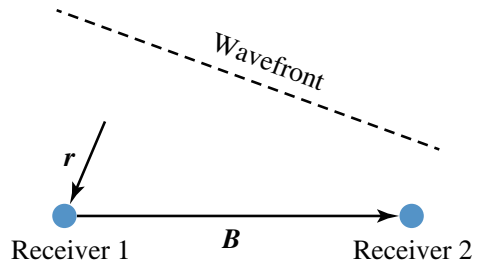

Figure 2 (Color online) Representation of the projection of the unitary wavefront vector upon the baseline vector.

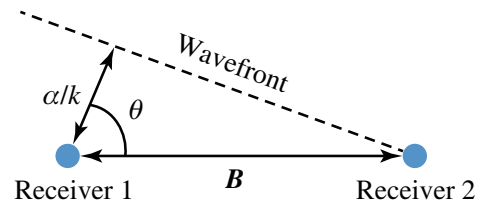

Figure 3 (Color online) Single baseline simplification.

where $T x(t)$ is the complex transmitted signal at the satellite antenna, $R x_{i}$ is the received signal at receiver $i, \alpha_{i}$ is the path delay phase, $\omega_{i}(t)$ is the additive white Gaussian noise added by the transmission path, and $t_{i}$ is the time delay from the satellite to the receiver $i$ and $A_{i}$ is the attenuation caused by the path losses.

The interferometric phase $\alpha_{12}$, defined as the difference between both path delays $\alpha_{1}$ and $\alpha_{2}$, can be retrieved by correlating both received signals during a delimited integration period. Only the zero lag component of the correlation is required for retrieving $\alpha_{12}$ from a punctual source, as described in (3).

$$
\left\langle R x_{1}, R x_{2}\right\rangle_{t=0}=\frac{1}{T} \sum_{0}^{k T} R x_{1}^{*}(n) R x_{2}(n)=E_{R} \mathrm{e}^{\left(\mathrm{j}\left(\alpha_{1}-\alpha_{2}\right)\right)},
$$

where $E_{R}$ is the received signal energy, $T$ is the time period, and $k$ is the sampling ratio. Notice that if the added samples are large enough, the noise can be substantially reduced by considering it greatly uncorrelated if the integration period is large enough. This principle enforces the use of correlation techniques instead of superposition techniques to reduce the noise on the interference signal.

This might lead to an unrestrainedly high integration time; however, the interferometer will provide a single value for each integration period, and hence a compromise must be established to retrieve a sufficiently sampled stream of data where noise is sufficiently uncorrelated, taking into account the relatives satellite-Earth motion.

Per se, the raw interferometric phase does not provide the position of the satellite; therefore, an orbital tracking model is required.

\subsection{Orbital tracking}

A mathematical model must be established in such a manner that it relates the three-dimensional position of the satellite with the raw data. At this point, two different models can be studied to determine the context in which they can provide value to our goals.

\subsubsection{Far-field approximation model}

In the far-field model, it is assumed that the distance between the signal emitter and the receiver is beyond the so-called Fraunhofer distance. Therefore, successive wavefronts are considered as parallel planes.

$$
d=\frac{2 D^{2}}{\lambda}
$$

where $D$ is the baseline length and $\lambda$ is the wavelength. As can be deduced from (4), this approximation may be considered valid for a geostationary emitter as long as the baseline is not larger than $650 \mathrm{~m}$ approximately. In the case where the interferometer is properly dimensioned according to this model, the geometry of the problem can be described in a vectorial model, as depicted in the following figures.

Therefore, the interferometric phase between each receiving pair is the geometrical delay of the wavefront between receivers expressed in phase units. This corresponds to the projection of the unitary range vector $\hat{r}$ upon the baseline vector $\boldsymbol{B}$.

$$
\alpha_{i j}=k\left(\hat{r} \cdot \overline{\boldsymbol{b})}=\frac{2 \pi}{\lambda}\left(B_{x} \sin (\theta) \cos (\phi)+B_{y} \sin (\theta) \sin (\phi)+B_{z} \cos (\theta)\right) .\right.
$$




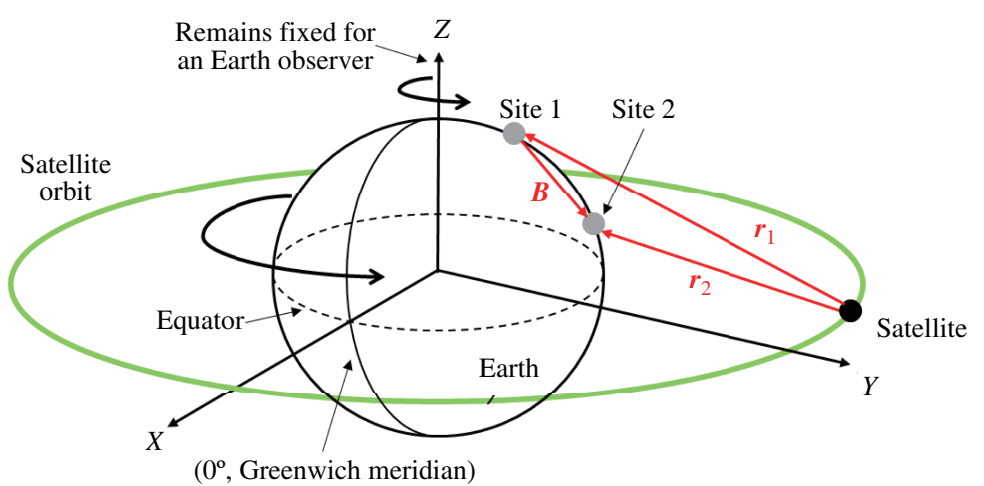

Figure 4 (Color online) Geometry of the three-dimensional Euclidean model.

Eq. (5) is typically used in direction finding interferometers $[9,10]$, as it provides a finite resolution only for angular data. On the other hand, the range is assumed to be infinite and cannot be determined.

In the single baseline case, an interesting result can be obtained by manipulating (5). If the $Z$ axis is defined in the same direction as the baseline, the following expression can be derived:

$$
\alpha_{i j}=\frac{2 \pi}{\lambda}(B \cos (\theta)) .
$$

This result is interesting, as it provides an algebraic solution from the arriving angle to the interferometric phase and vice versa. Thus, a direct tracking method is provided.

$$
\theta=\arccos \left(\frac{\lambda}{2 \pi} \frac{\alpha_{i j}}{B}\right)
$$

By using (8), the tracking resolution can be algebraically obtained by considering the effect of a threshold phase variation $\sigma_{\alpha}$ in the angle of arrival.

$$
\delta \theta=\frac{\lambda}{2 \pi} \frac{\sigma_{\alpha}}{B \sin (\theta)} .
$$

In conclusion, while this mathematical model provides an approximate result and is limited by the interferometer dimensions, it provides intuitive information about the performance of the device.

\subsubsection{Euclidean model}

From a strictly geometrical point of view, allowing for no assumptions or approximations, the signal delay between both receivers is equal to the wave number multiplied by the signal path difference described by the euclidean distance.

$$
\alpha_{12}=\frac{2 \pi}{\lambda}\left(\left|\boldsymbol{r}_{1}\right|-\left|\boldsymbol{r}_{2}\right|\right)
$$

This mathematical model is valid regardless of the baseline dimensions and it provides a fully relational orbital observable, where the raw data depends on the three orthogonal axes of space. While this approach seems appropriate, the lack of an algebraic expression in the form of $[x, y, z]=f\left(\alpha_{i j}\right)$ forces the use of numerical methods to achieve satellite tracking. This fact precludes the possibility to provide mathematically simple expressions to relate each parameter.

Therefore, the Euclidean model is used to perform satellite tracking; however, this model would not allow to determine algebraically how each parameter (i.e., baseline length) affects the quality of the retrieved orbit.

By using this model, the line separating orbital tracking and orbital determination becomes blurred as the operation of tracking is embedded in the determination method instead of performing both steps separately as revealed in the following section. 


\subsection{Orbit determination}

Orbit determination involves different methods and techniques adopted to determine the satellite orbit from a collection of observation data provided by orbital tracking, for instance, a set of ranges, angles, velocities, etc.

In the context of a ground interferometer system, such observation data are the interferometric phase observations. These observations cannot provide orbital elements by themselves because no analytical method is available in the literature that connects interferometric phase observations to orbital elements in a straightforward way. One may transform the interferometric phase observations into angular observations and find orbital elements by using Laplaces method, Gauss method, etc. [11]; however, such a transformation could considerably complicate the problem and the accuracy of these methods would not be satisfactory.

On the other hand, one can use differential correction techniques. These techniques require iterations or incremental updates to the state ${ }^{1)}$ that improve the accuracy of orbit determination by using the methods mentioned before. To obtain orbital elements from interferometric phase observations, leastsquares techniques are proposed.

Least-squares techniques make use of all the data available to improve the determination of an approximate initial $s_{a t}{ }^{2)}, \bar{x}$. They are defined as an optimization problem that fits the measurements to an appropriate mathematical model ${ }^{3)}$, minimizing the sum of the squares of the residuals. The residuals will be the difference in the actual observations and those obtained using the state vector solution. Thus, defining the residuals as

$$
\bar{r}=y_{0}-y_{c},
$$

where $y_{0}$ are the observed values of the dependent variable and $y_{c}$ are the computed values of the dependent variable, the least-squares criterion (for $N$ observations) satisfies the minimization of the following expression:

$$
J=\sum_{i=1}^{N}{\overline{r_{i}}}^{2} .
$$

The complete formulation of the least-squares technique for orbit determination can be found in [11] providing the following equation:

$$
\delta \overline{\boldsymbol{x}}=\left(\boldsymbol{A}^{\mathrm{T}} \boldsymbol{W} \boldsymbol{A}\right)^{-1} \boldsymbol{A}^{\mathrm{T}} \boldsymbol{W} \tilde{\boldsymbol{b}}
$$

where $\delta \overline{\boldsymbol{x}}$ is the estimated correction to the state, $\boldsymbol{A}$ is the partial-derivative matrix, $\boldsymbol{W}$ is the weighting matrix, and $\tilde{\boldsymbol{b}}$ is the residual matrix. The resulting $\delta \overline{\boldsymbol{x}}$ value must be added to the initial state and the least-squares algorithm will iterate again by using this new initial state value until a convergence criterion is reached ${ }^{4)}$. Once the convergence criterion is achieved, the improved initial state will be provided by the algorithm. This initial state can then be used to obtain the orbital elements..

\section{Prototype implementation}

A two-element interferometer has been implemented to carry out a preliminary test of concept. A constellation of geostationary television broadcast satellites has been chosen as the emitters of opportunity. The constellation properties are presented in Table 1.

The aim of the prototype is to capture the signal from this constellation and to perform appropriate interferometric processing. For this purpose, two coherent $\mathrm{Ku}$ band receivers are placed on the rooftop of the UPC Campus Nord D3 building at known locations, as depicted in Figure 5.

1) The state of a satellite in space is defined by six quantities that can be called either a state vector (position and velocity vectors) or an element set (a collection of scalar magnitudes and angular representations of the orbit).

2) This initial state can be obtained, for example, from the known longitude coordinates of the satellite at one specific epoch of time.

3) In the case under study, the mathematical models involved in the operation are the Euclidean model for orbital tracking and Kepler's laws for orbit determination.

4) Some methods to determine the criterion of convergence are discussed by Vallado in [11]. 
Table 1 Satellite constellation properties

\begin{tabular}{cc}
\hline Orbital slot & $19.2^{\circ} \mathrm{E}$ \\
Number of satellites & 4 \\
Operator & SES Astra \\
Satellite names & ASTRA $1 \mathrm{KR} / 1 \mathrm{~L} / 1 \mathrm{M} / 1 \mathrm{~N}$ \\
Frequency band & $10.6-12.6 \mathrm{GHz}$ \\
Average channel bandwidth & $30 \mathrm{MHz}$ \\
Channel modulation & QPSK or 8 -PSK \\
\hline
\end{tabular}

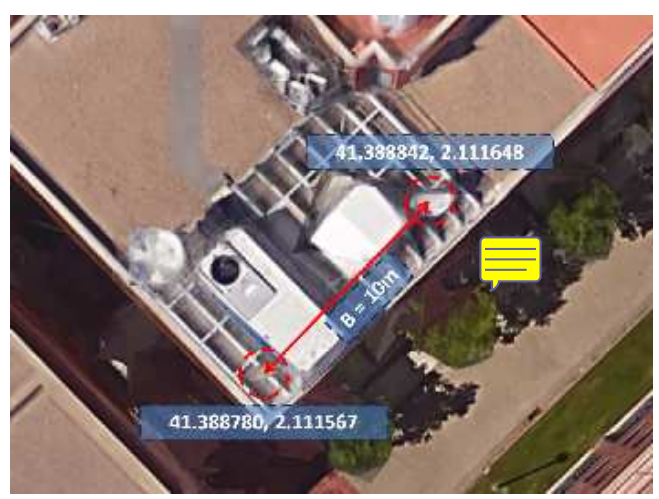

Figure 5 (Color online) Zenith view of the receiver locations at the UPC Campus Nord D3 building.

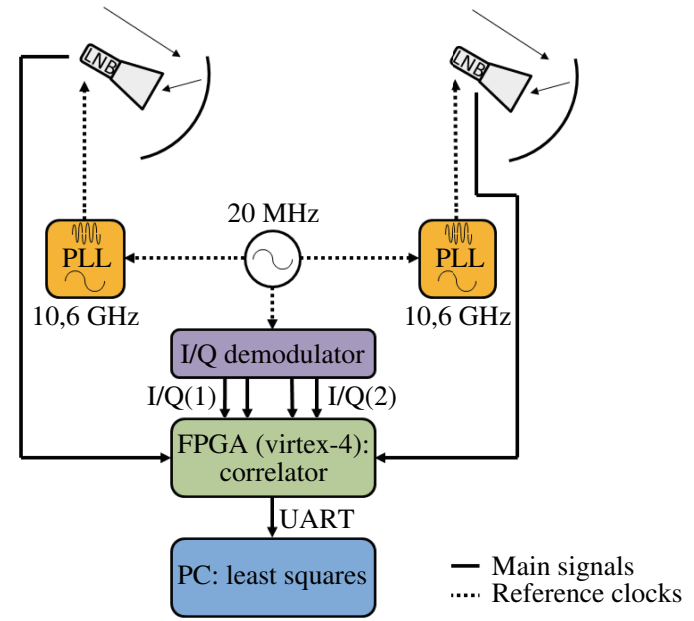

Figure 6 (Color online) Conceptual block diagram of the interferometer depicting the hardware performing the full operation

\subsection{Ku band receiver}

By using a low-noise down-converter block (LNB) integrated in the antenna feeder, a multichannel IF 1-2 GHz signal can be guided to the receiver with tolerable attenuation by means of low-cost coaxial cables. Implementing all receiver steps with common local oscillators is strictly necessary to ensure coherency. Thus, the phase difference of the signals arriving at both the receivers is preserved. In the case under study, both LNB internal oscillators have been replaced by external ports fed by a common PLL reference oscillator.

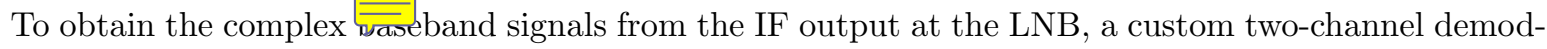
ulator has been designed. The demodulator output bandwidth is $40 \mathrm{MHz}$ for a total of 2-GHz bandwidth available at the IF stage. Corresponding to one of the multiplexed DVB-S channels. Since each individual channel is emitted by one of the four satellites in the constellation, by selecting the channel properly, the actual satellite being tracked can be chosen. 


\subsection{Digital signal processing}

\subsubsection{Complex correlation}

The correlation operation between both complex signals is performed by a Virtex-4 FPGA as a low-level operation. To ease the task of both correlating and digitizing the signal, an FPGA digital port has been used as a two-level quantization device, performing the analog to digital conversion and allowing for the correlator to be implemented as a simple XOR network.

The loss of information caused by poor quantization can be overcome by using the Van Vleck correction [12], which establishes a mathematical relationship (13) between the ideal correlation $\rho$ of two analog signals and the correlation of the two-level digitized signals $\hat{\rho}$.

$$
\rho=\sin \left(\frac{\pi}{2} \hat{\rho}\right)
$$

\subsubsection{Orbit determination}

Let us obtain the orbital elements from the interferometric phase observations retrieved by following the steps mentioned in the previous section. Results have shown that, by using only one baseline, the orbital parameters achieved after least-squares processing are more reliable when the observation data are acquired for a long time, e.g., two days. Thus, the possible satellite orbits that fulfill such observation data are more accurate.

Another major issue to be considered before implementing the least-squares algorithm is the phase ambiguity. The interferometric phase observations are given in the interval $[0,2 \pi)$. Unless we add the integer number of phase cycles lost between the satellite and the site antennas, multiple satellite orbits will satisfy such observation data, and therefore the least-squares algorithm will not converge. As dealing with phase ambiguity is not the aim of this work, it has been simplified as follows. Given a TLE ${ }^{5)}$ of the geostationary satellite orbit [13] used as the reference orbit, the integer number of phase cycles has been computed by simulating the virtual interferometric phase produced by the motion described in the TLE. By this trick, one can find an approximate number of phase cycles not far from the real one. It is important to note that this technique is only suitable in case of very short baselines, in the order of tenths of meters, as it is the case here. An alternative ambiguity resolution technique should be studied for larger baselines.

Once the interferometric phase observations are retrieved taking into account all the points mentioned above, the least-squares algorithm may be applied. Thus, the approximate initial state vector, $\bar{x}$, will be refined by means of all observation data collected during several hours by the ground-based interferometer system. After the implementation of the least-squares algorithm, a new state vector is achieved that can be transformed into an element set to compare all its magnitudes to the reference TLE orbit. In this way, we can obtain a first evaluation of how the ground-based interferometer system works by using a single short baseline.

As the aim of this work is to demonstrate a successful proof of concept rather than a high-accuracy device, we implemented the least-squares algorithm by using a two-body propagator. Thus, orbital perturbations such as the force exerted by the Earths equatorial bulge, the solar radiation pressure, etc. have been neglected, and therefore the classical orbital elements have been considered as constants during the entire orbital determination period.

\section{Results analysis}

In this section, we present the final results achieved from the previously described prototype. First of all, the complete stream of acquired raw data will be presented. Second, the retrieved orbit will be analyzed

5) A two-line element set is a data format encoding a list of orbital elements of an Earth-orbiting object for a given point in time. 
Table 2 Acquisition parameters

\begin{tabular}{cc}
\hline Satellite & ASTRA 1N \\
Central frequency & $12.051 \mathrm{GHz}$ \\
Bandwidth & $40 \mathrm{MHz}$ \\
Integration time & $1 \mathrm{~s}$ \\
Acquisition start time & $05 / 07 / 20160: 56: 29(\mathrm{GMST}+2)$ \\
Acquisition end time & $11 / 07 / 20167: 56: 38(\mathrm{GMST}+2)$ \\
\hline
\end{tabular}

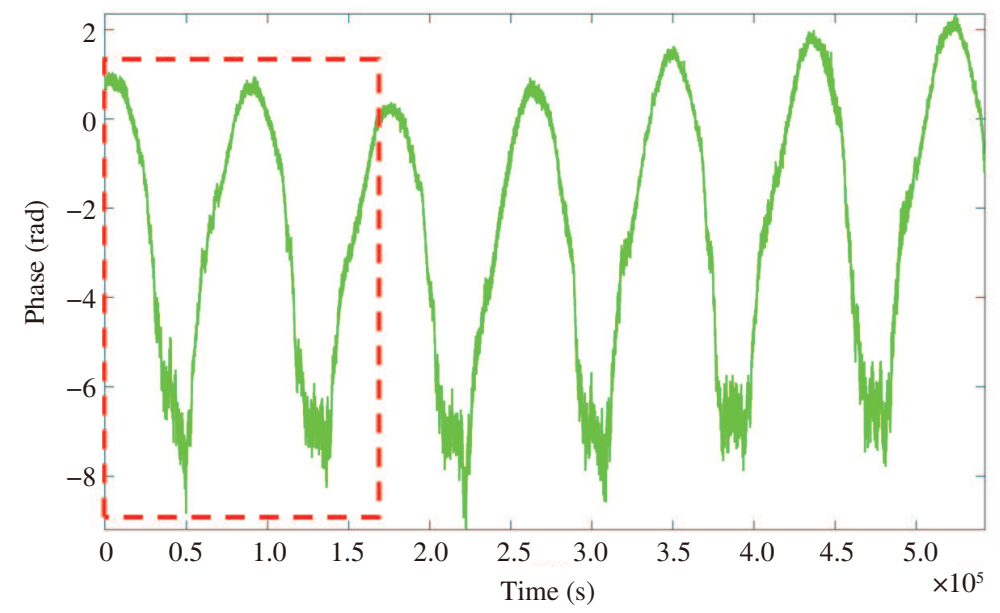

Figure 7 (Color online) Stream of unwrapped raw data acquired during 6 days. One sample per second. The highlighted area represents the data used during the orbital determination procedure.

and compared to the public orbital dataset. Finally, a set of conclusions from the prototype results will be discussed. Table 2 summarizes the configuration parameters used during the data acquisition campaign.

\subsection{Raw data}

Figure 7 depicts the full six-day stream of interferometric phase retrieved by the prototype system. These data have been properly unwrapped in a progressive way from the first sample. As seen in the figure, the entire signal describes a sine-like waveform featuring a period of a sidereal day, as expected for a geostationary orbit. The amplitude of the signal $(\sim 10 \mathrm{rad})$ is relatively small owing to the short baseline separating the two receivers.

The signal-to-noise ratio presents a cyclic behavior where the negative lobes (daytime) are considerably noisier than the positive lobes (night time). Apart from the known atmospheric effects present during daytime [14], sun exposure of the cables connecting the two receivers might cause such behavior.

From the total of days available, only the orbit during the first two days (highlighted area) will be retrieved as an example.

\subsection{Retrieved orbit}

The following tables present a comparison between the initial state vector obtained by the TLE set ptums hed by the Joint Functional Component Command for Space (a component of the US Strategic Command), and the state vector retrieved by the least-squares algorithm from the unwrapped, full-cycle raw data. Both state vectors are expressed in an ECI coordinate frame ${ }^{6)}$.

As seen from the tables, the discrepancy between both state vectors is not excessively large, considering the use of a single 10-m baseline. This fact can be more graphically appreciated in Figure 8, where both

6) The Earth-centered inertial coordinate frame originates at the center of the Earth and is generically designated with the letters $I J K$. The fundamental plane contains the Earth's equator. The $I$ axis points towards the vernal equinox, the $J$ axis is $90 o$ to the east in the equatorial plane, and the $K$ axis extends through the North Pole. 
Fuster R M, et al. Sci China Inf Sci January 2017 Vol. 60 xxxxxx:9

Table 3 Comparison between the TLE state vector and the estimated state vector (position components)

\begin{tabular}{cccc}
\hline ECI coordinates & $r_{x}(\mathrm{~km})$ & $r_{y}(\mathrm{~km})$ & $r_{z}(\mathrm{~km})$ \\
\hline SV propagated from TLE & 12078.647 & -40334.464 & -33.470 \\
SV retrieved from interferometer & 12069.240 & -40381.556 & -53.737 \\
Difference & 9.406 & 47.093 & 20.267 \\
\hline
\end{tabular}

Table 4 Comparison between the TLE state vector and the estimated state vector (velocity components)

\begin{tabular}{cccc}
\hline ECI coordinates & $v_{x}(\mathrm{~km} / \mathrm{s})$ & $v_{y}(\mathrm{~km} / \mathrm{s})$ & $v_{z}(\mathrm{~km} / \mathrm{s})$ \\
\hline SV propagated from TLE & 2.947 & 0.883 & 0.002 \\
SV retrieved from interferometer & 2.944 & 0.881 & 0.012 \\
Difference & 0.003 & 0.002 & -0.010 \\
\hline
\end{tabular}
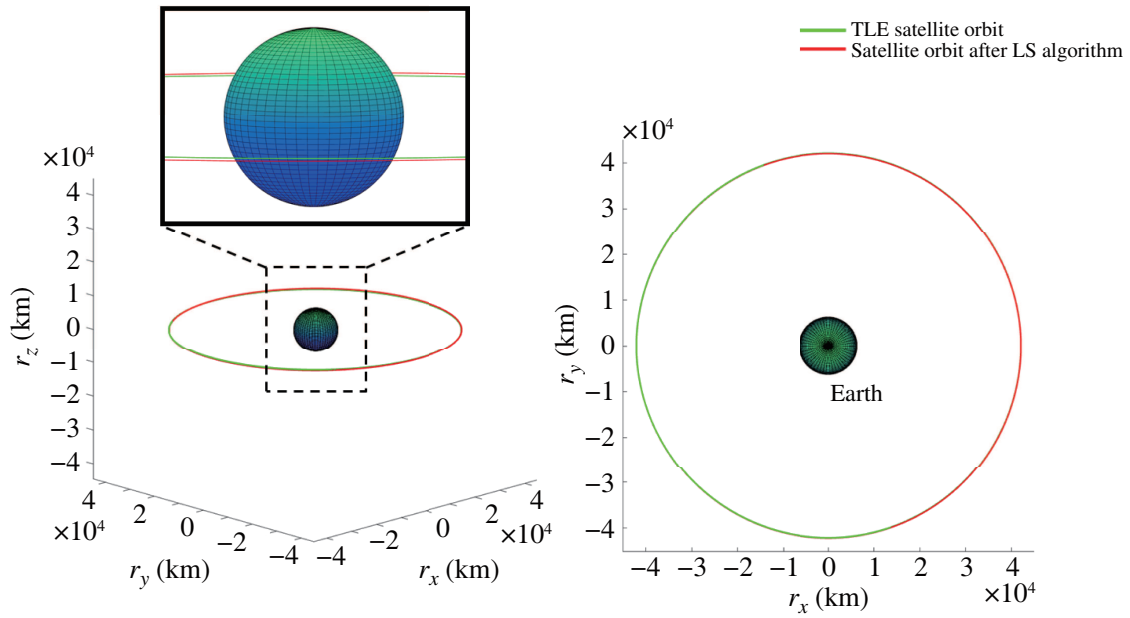

Figure 8 (Color online) Propagated orbits from the initial TLE state vector (green) and the least-squares-processed interferometric phase (red). 48 hours period.

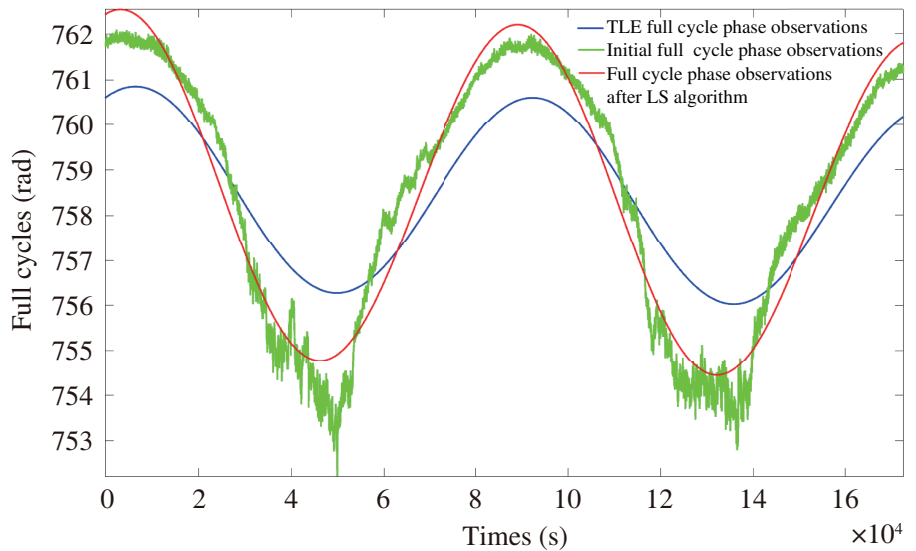

Figure 9 (Color online) Representation of the acquired interferometric phases (green) and the simulated phases from the TLE (blue) and the least-squares filter output (red).

state vectors have been propagated in time and plotted alongside.

Figure 9 depicts a comparison among different interferometric phases. In this way, the full-cycle interferometric phases simulated from the TLE propagation, the unwrapped, full-cycle raw data used during the signal processing, and the full-cycle interferometric phases simulated from the propagation of the state vector after the least-squares algorithm implementation are plotted. A common pattern can be appreciated among the three curves.

A close look at Table 5 manifests some discrepancies between the expected orbital elements and the 
Fuster R M, et al. Sci China Inf Sci January 2017 Vol. 60 xxxxxx:10

Table 5 Comparison between the TLE-published orbital elements (left) and the estimated orbital elements (right)

\begin{tabular}{cccc}
\hline Orbital element & Symbol & From TLE & From interferometer \\
\hline Inclination & $i\left(^{\circ}\right)$ & 0.056 & 0.228 \\
Right ascension of the ascending node & $\Omega\left(^{\circ}\right)$ & 341.4 & 305.3 \\
Eccentricity & $e$ & $4.51 \times 10^{-4}$ & $14.9 \times 10^{-4}$ \\
Argument of perigee & $\omega\left(^{\circ}\right)$ & 101.0 & 144.3 \\
Mean anomaly & $M\left(^{\circ}\right)$ & 204.3 & 128.1 \\
Mean motion & $n(\mathrm{rev} /$ day $)$ & 1.003 & 1.003 \\
\hline
\end{tabular}

orbital elements obtained. The discrepancies can be explained by different factors:

- Baseline length: As exposed in the far-field approximation model for accuracy by expression (8), an inverse proportionality ratio exists between the interferometric phase accuracy and the baseline. Therefore, it can be concluded that a 10-m baseline is not large enough to provide an accurate satellite orbit. Recent simulations conclude that at least a few kilometer baselines are required to achieve centimetric accuracy.However, atmospheric phase perturbations must be experimentally analyzed in this case.

- Basel dimensionality: A single baseline is able to provide sensitivity to the satellite displacement only on the baseline direction. Therefore, the setup is poorly dimensioned for retrieving a three-dimensional motion. As a matter of fact, being able to retrieve a geosynchronous orbit using a single baseline, even at this accuracy levels, is rather surprising. A setup consisting of multiple orthogonal baselines would provide sensitivity in multiple dimensions, enhancing the resulting accuracy.

- Daytime noise: Given the low sensitivity of the system, the visible noise on the phase during daytime generates perturbations on the resulting orbital model, and would have much less impact for a larger baseline configuration. This noise can be reduced by means of a calibration loop that monitors unwanted phase variations.

Finally, approximate dimensioning of a future system must be studied to determine its scalability to provide an accurate orbit. According to (8), if the actual prototype accuracy is tenths of kilometers, the prototype should be enlarged to tenths of kilometers for a single baseline to obtain accuracy in the order of meters, and this is not an infeasible size for a ground-based interferometer. In fact, numerical simulations developed by using the Euclidean model show that, for a three-baseline interferometer, submeter accuracy could be achieved with dimensions less than $1 \mathrm{~km}$, since the problem can be better stated in the case of three dimensions.

\section{Conclusion}

This work shows that a ground-based interferometer is able to provide observables suitable for precise orbit determination required in GeoSAR missions.

The least-squares algorithm, typically designed to retrieve orbits by using range and range rate as input data, can be properly modified by means of the Euclidean model to retrieve orbital elements from the interferometric phase. Nevertheless, the use of a single baseline affects the performance of the algorithm by obstructing the convergence of the optimization unless a large period of time is used.

Exploiting the interferometric phase provides accuracy in orders of magnitude better than that of systems using time domain data, since the phase is more sensitive to motion. This fact offers potential accuracies that would be able to be used in GeoSAR missions. Even in the case where the required millimetric accuracy cannot be provided, further autofocus processing methods can be applied to retrieve the SAR image.

The interferometer does not require any particular transmission code, and therefore could be easily fitted in a GeoSAR environment, either by using the actual radar signal or by adding a dedicated beacon on the actual satellite. Furthermore, since this technique is not based on ground calibrators, the interferometer can be located anywhere as long as the satellite signal is received, regardless of the location actually being imaged. 
Fuster R M, et al. Sci China Inf Sci January 2017 Vol. 60 xxxxxx:11

This prototype will be enhanced in the near future on the basis of upgrades suggested for this work.

Acknowledgements This work has been financed by the Spanish Science, Research and Innovation Plan (MINECO) with Project Code TIN2014-55413-C2-1-P.

Conflict of interest The authors declare that they have no conflict of interest.

\section{References}

1 Hu C, Long $\mathrm{T}$, Zeng $\mathrm{T}$, et al. The accurate focusing and resolution analysis method in geosynchronous SAR. IEEE Trans Geosci Remote Sens, 2011, 49: 3548-3563

2 Ruiz-Rodon J, Broquetas A, Makhoul E, et al. Nearly zero inclination geosynchronous SAR mission analysis with long integration time for Earth observation. IEEE Trans Geosci Remote Sens, 2014, 52: 6379-6391

3 Monti Guarnieri A, Hu C. Geosynchronous and geostationary SAR: face to face comparison. In: Proceedings of 11th European Conference on Synthetic Aperture Radar, Hamburg, 2016. 1-4

4 Ruiz-Rodon J, Broquetas A, Monti Guarnieri A, et al. Geosyncrhonous SAR focusing with atmospheric phase screen retrieval and compensation. IEEE Trans Geosci Remote Sens, 2013, 51: 4397-4404

$5 \mathrm{Hu}$ C, Li Y H, Dong X C, et al. Performance analysis of L-band geosynchronous SAR imaging in the presence of ionospheric scintillation. IEEE Trans Geosci Remote Sens, 2017, 55: 159-172

6 Martin Fuster R, Fernández Usón M, Casado Blanco D, et al. Proposed satellite position determination systems and techniques for Geostationary Synthetic Aperture Radar. Eur J Remot Sens, 2016, 49: 735-744

7 Lengyel B A. A Michelson-type interferometer for microwave measurements. Proc IRE, 1949, 37: 1242-1244

8 Holler C M, Jones M E, Taylor A C, et al. A 220-GHz analog lag correlator for radio interferometry. IEEE Trans Instrum Meas, 2012, 61: 2253-2261

9 Qin L, Jia K X, He Z S. Performance analysis of correlative interferometer direction finder using cosine function. In: Proceedings of International Symposium on Intelligent Signal Processing and Communication Systems, Chengdu, 2010. $1-4$

10 Duncan J W. The effect of ground reflections and scattering on an interferometer direction finder. IEEE Trans Aerosp Electron Syst, 1967, AES-3: 922-932

11 Vallado D A. Fundamentals of Astrodynamics and Applications. Hawthorne: Microcosm Press, 2013

12 Van Vleck J H, Middleton D. The spectrum of clipped noise. Proc IEEE, 1966, 54: 2-19

13 National Aeronautics and Space Administration. Definition of two-line element set coordinate system. http: //spaceflight.nasa.gov/realdata/sightings/SSapplications/Post/JavaSSOP/SSOP_Help/tle_def.html

14 Morabito D D, D'Addario L R, Acosta R J, et al. Tropospheric delay statistics measured by two site test interferometers at Goldstone, California. Radio Sci, 2013, 48: 729-738 